\title{
Applied Color Sensor Based Solution for Sorting in Food Industry Processing
}

\author{
Mohamed Amine Alaya', Zsolt Tóth¹, Attila Géczy² \\ ${ }^{1}$ Institute of Information Science, Faculty of Mechanical Engineering and Informatics, University of Miskolc, \\ H-3515 Miskolc-Egyetemváros, Hungary \\ 2 Department of Electronics Technology, Faculty of Electrical Engineering and Informatics, Budapest University of Technology \\ and Economics, H-1111 Budapest, Egry József u. 18., Hungary \\ * Corresponding author, e-mail: amine.alaya@ett.bme.hu
}

Received: 31 August 2018, Accepted: 30 October 2018, Published online: 22 January 2019

\begin{abstract}
The market of food products is growing significantly, and this affects commercialization and potential consumption aspects. Sorting is mandatory from efficiency and precision approaches in production. Our paper presents a simple and cost effective solution for presenting a sorter automaton (self-operating machine), with applied sensorics. The idea is to apply a color sensor (TCS3200) which analyzes any type of product by its color and sort it for the production line with the help of the servo- and stepper motors. The system will be controlled by a simple card computer. The color sensor detects the frequency of the wavelength reflected from the product and give the information to the processing logic, which translates the 3D RGB cube based data. Then the logic makes a conversion to HSL (hue, saturation, lightness) derived in degrees $\left[0^{\circ}, 360^{\circ}\right.$. The investigation of the machine has been implemented for an exemplary colored candy sorting. The resulting colors of the analyzed samples are selected in a specific calibration set of numbered color ranges. The proposed automaton and its validation are presented in details.
\end{abstract}

\section{Keywords}

applied sensorics, food industry, color sensor, automation

\section{Introduction}

Color, shape and other quality based sorting is a persistent problem in the food industry [1, 2]. Such technology is needed in order to sort fruits and vegetables, such as olives [3], dates [4], tomatoes [5], peppers [6] canned peaches, grains, crops, or manufactured-processed food products [7], with the objective of saving time and efficient sorting according to different parameters. In fact, sorting vegetables or seeds is still undeveloped and performed manually in some countries [8]. Manual sorting costs the underdeveloped industries a considerable amount of time and energy, and reduces effectivity and precision. Color detection is a major issue in this field. Granules [8], liquids $[9,10]$ or powders, likewise other products, such as minerals, glasses, plastics or even clothes are investigated and detected regarding color characteristics.

The main idea of our work was inspired by the need of industries to classify and store products by color in a cheap and efficient way. The project aims to improve the productivity and to advance the sorting processes. As a case study, the paper gives a proper insight to what is needed for the solution of a given process, and it focuses both the electronics and industrial informatics aspecst of such technologies.

The basic function of our solution is to sort coated chocolate candies by color. For this, the applied use of color sensorics is considered. The method can be extended to crops sorting [7] and other previously mentioned food types as well.

\subsection{Sensors in food industry}

Evaluation of food with various sensors started to develop between 1930-1950; since then food industry improved its automated approaches and it is still refining already developed methodologies [11]. The refinement can point to better quality aspects with state of the art hardware and software configurations, and improvement may also point to cheaper and more cost effective solutions.

Color sensors and sensor based setups were used in the food industry in various applications recently [12-14]. 
As an interesting application, computer vision and simple sensor kit was used for Salmon Fillet qualification [15] with the help of RGB to CIE Lab conversion [16]. Recently a software enabled method was used to investigate with camera based colorimetry approaches [17]. Sensor based colorimetry was also used recently to analyze toxic materials in fruit samples [18]. According to the recent literature, cheap and cost effective solutions still need further investigations.

\subsection{Color measurement}

The majority of the food industries use two main color measurement techniques (Colorimetry and Spectrophotometry). Colorimetry is a technique based on measuring the three primary colors observed by human capabilities (RGB). Food industries are using this type of colorimetric sensors to define different factors such as the depletion and corruption for respect of storage and shelf life time. However, this technique cannot detect the secondary and tertiary colors individually which makes its efficency limited. Spectrophotometry can measure the different wavelengths of visible light, 400 to $700 \mathrm{~nm}$, either this length is given by the spectral reflectance or by the transmittance of an object. For this reason spectrometry has gained ground in the food industry, which is used to measure and standardize color. This technology is reliable to define the characteristics of material color (especially in cans, drinks, etc.), however requires more complex hardware setups.

A typical color sensor is based either on Photo-emissive Cells, Photo-conductive Cells, Photo-voltaic Cells or Photo-junction Devices. Nevertheless, in this study we focus solely on a Photo-junction type device which measure reflection of red, green and blue light sources. It is possible for several advanced sensors to obtain Hue colors. In this project, the used sensor is working according the aforementioned principles, however the sensor core has embedded filters for the three basic colors - this way, the light source should be white.

Summing up, our aim is to find an efficient way to sort the maximum number of products with minimum time, and an accuracy higher than $90 \%$, only by using a simple system and the cheapest available devices. While focusing on the optimization for cost and simplicity, our solution points to novel use of card computers and widely available commercial sensors to show the efficiency and potential of these widely available products. Our approach is also compatible with IoT aspirations and Industry 4.0 aspects [19].

\section{Experimental}

\subsection{The color sensor}

The color sensor which is used in our configuration is the TCS3200 sensor module from TAOS (see Fig. 1). In this module silicon photodiodes and a current-to-frequency converters are integrated into a CMOS circuit. The converters are aligned in a $8 \times 8$ matrix. 16 photodiodes have red filters, 16 photodiodes have blue filters, 16 photodiodes have green ones, and 16 photodiodes have none. The inputs can be chosen so that respectively one of these colors can be detected by the sensor, and in the output, the signal is set for a basic square wave (50\% duty cycle) along with frequency directly equivalent to light intensity of the chosen color. In our approach, this sensor is interfaced with a lens, and a set of white LEDs which are required for the best feedback. The setup was used with the support board AXE045 from PIXACE. The light sources are configured on the module, to enable adequate reading on the samples.

\subsection{General setup}

The overall electrical system is based on the color sensor, which is connected to the servo motor. The color sensor and the servo are connected to an Arduino single board microcontroller (simple card computer based on a microcontroller), which is the central logic of the system (see Fig. 2).

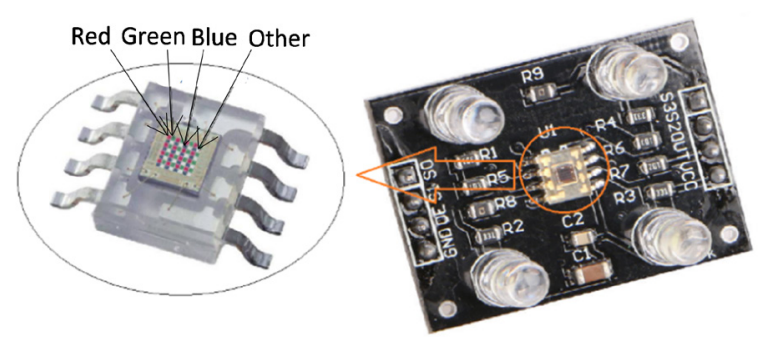

Fig. 1 Photodiodes of the sensor TCS3200

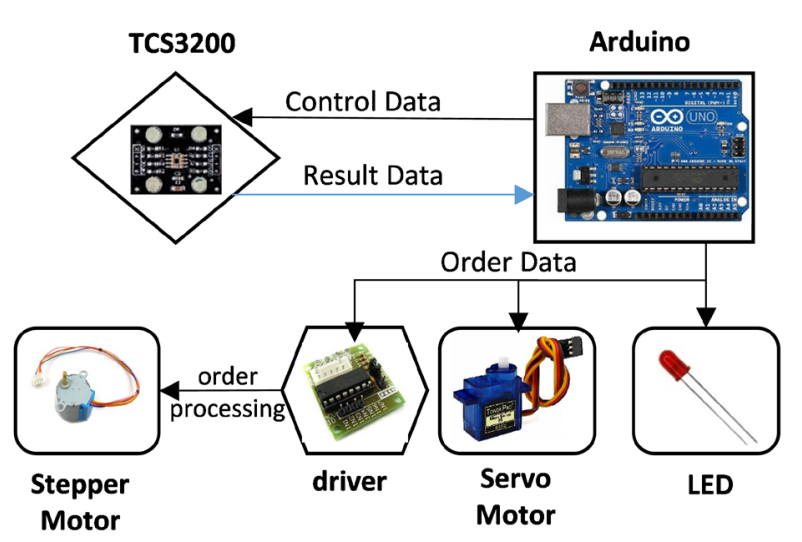

Fig. 2 Electrical overview of the system 
The stepper motor recive the signal from the Arduino, based on the color sensor signal to allow the first movement of the item. Unipolar steppers and bipolar stepper motors are the most basic and widespread kind of stepper motors, and based on the project requirmenets, the bipolar stepper motor suits our needs better because it can provide the 8 movement steps (positions). The Arduino provided a control singnal to the stepper motor and power is provided to the stepper by an external power supply. The system is taking the inspiration from the work of Goudet [20], and improves upon many points, including the more accessible card microcontroller setup.

\subsection{Sensor setting}

The TCS3200 is a color light-to-frequency converter, which provide square wave with frequency ouput proprotial directly to the light intensity. Each color is represented by the photodiode filters that are connected in parallel. By changing the input signal bits iS2 and iS3 (shown in Table 1) the activatied photodiodes receive the intensity of the color. After the activation of the 4 group of photodiodes, the result will analysed finally by the Arduino.

\subsection{The setup of the State Machine}

The finite-state machine (FSA) used is presented in Fig. 3. And Table 2 describes the steps of the state machine according to Fig. 3. The entry action in state S0 starts a motor that opens the path of the candy then the sensor adjusts the color, the entry action in state S6 starts a servo in the different direction for classification of the candy. States S0 and S6 stop the motor when there is no more candy left, and this state can be provided to other interface machines to sign the end of operation.

The general operation algorhithm of the embedded software is shown by the flowchart presented in Fig. 4. The system operation starts after activating the elements of the system (Arduino, Servo, Stepper, Sensor etc.) and the rest of the instruments (color detector); then the Arduino loop algorithm initializes. Then it starts checking the item postion (avaliability) at the color sensor to give the signal to the stepper motor, so that the stepper motor will move the items to the next step regardless of its color. But by this step the items are ready in a queue for actual color testing by the color sensor. Then the sensor will read the values of the red, green and blue intensities (RGB) and send the measurements to the Arduino to convert it from the RGB to HSL (hue, saturation, lightness) range. If the Arduino recognizes that there are no items after determining time
Table 1 Selectable input options

\begin{tabular}{ccc}
\hline iS2 & iS3 & Photodiodeactivation \\
\hline L & L & Red \\
L & H & Blue \\
H & L & Clear (no filter) \\
H & H & Green \\
\hline
\end{tabular}

Table 2 States of the process

\begin{tabular}{lc}
\hline Steps & State process \\
\hline S0 & $\begin{array}{c}\text { initialization } \\
\text { S1 }\end{array}$ \\
S2 & move the stepper motor to put the candy under the color \\
sensor
\end{tabular}

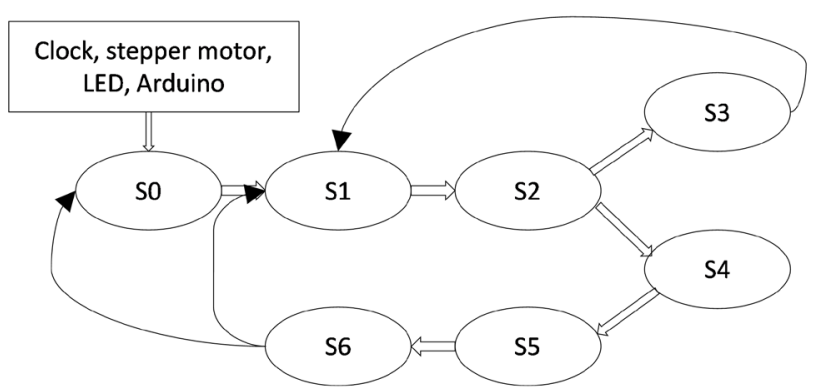

Fig. 3 Finite-state machine (FSA) define states

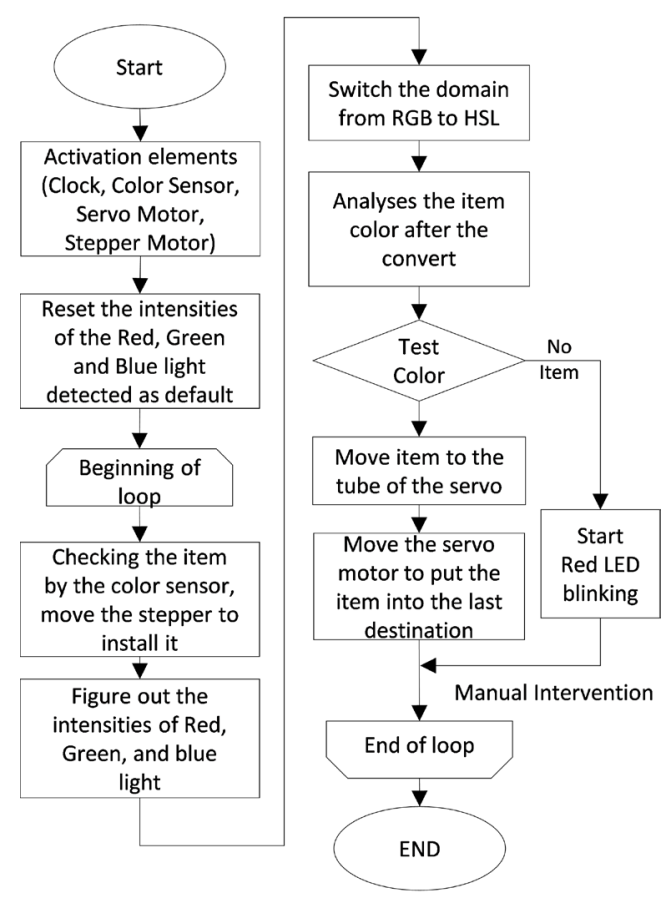

Fig. 4 General operation of the embedded software 
then the red LED will start blinking. If there is an item to be measured, the Arduino will find the range of the color and send the order to the motor, to sort the item to the right direction. As shown in the flow chart the loop ended if the items (candies) ended, or the system stopped manually.

\subsection{Sensor setup}

Color sensors measurments provide all the colors frequencies but a conflict exist due to the measurement error that effect on the measurments of short range color degrees; for example the orange color degree range is short $\left[8^{\circ}, 1^{\circ}\right]$ while the blue range $\left[180^{\circ}, 309^{\circ}\right]$ is large, hence if an error is measured near to the limits then a conflict of reading may happen. As a consequence, the system is more susceptible for mistakes in classification at the shorter ranges. It is also difficult to detect the yellow color, because samples in near-yellow has higher ability to reflect the light. This case can be confusing especially with the orange color. On the other hand, the green and the blue colors are easy to detect because their frequencies are totally far from the other colors, therefore, we found that there were no chance of error in detection.

The conversion of the RGB models is performed via HSL (hue, saturation, lightness) and HSV (hue, saturation, value) approach, because it just depends on the RGB space and gamma correction, focus on the three primaries (Red, Green and Blue). Each single RGB device contain individual HSL and HSV color spaces.

To determine the result, we need to go step by step through the following mathematic formulas. This starts with determining the difference between the largest and smallest values between $R, G$, or $B$. We determine the values of the maximum and minimum components such as $M$ and $m$ (the maximum is the highest value of the three primary result of red, green and blue, and the minimum is the smallest value), respectively:

$$
\begin{aligned}
& M A X=\max (R, G, B) ; \\
& \min =\min (R, G, B) ; \\
& \text { Chroma }=M A X-\min .
\end{aligned}
$$

The hue is the distance ratio around the edge of the hexagonal corridor across the projected point, which is initially measured in the interval $[0,1]$, but it will be mentioned in degrees $\left[0^{\circ}, 360^{\circ}\right]$ (see Fig. 5 and Fig. 6). For points that appear on the parent at the color level (such as, gray), the color is unknown.

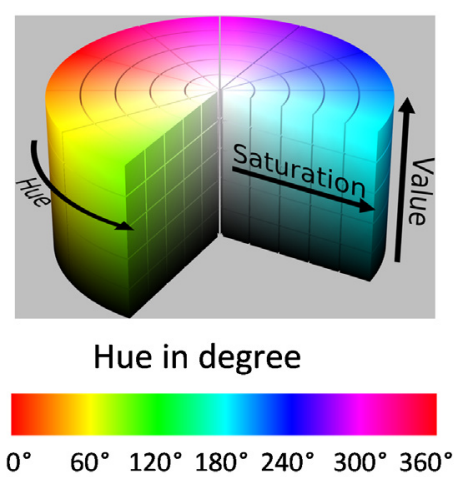

Fig. 5 The Hue greation

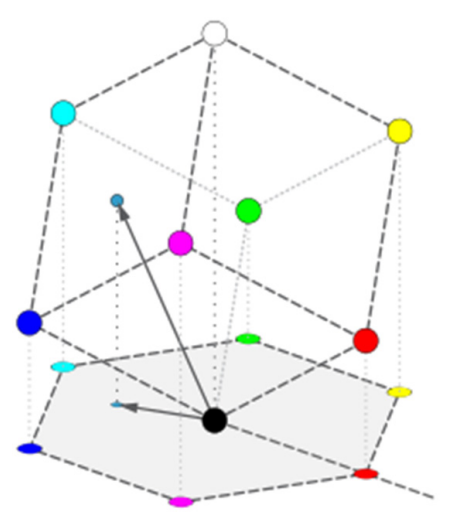

Fig. 6 Reflection of the RGB color cube on one plan

Mathematically, this definition of hue is written in the following manner:

$$
H^{\prime}=\left\{\begin{array}{l}
\text { undefined, } C=0 \\
\frac{G-B}{C} \bmod 6, M=R \\
\frac{G-B}{C}+2, M=G \\
\frac{R-G}{C}+4, M=B
\end{array}\right.
$$

hue $=60 \times \mathrm{H}^{\prime}$;

we can obtain the model-builders of HSL and HSV by taking the RGB cube model [21] $R, G, B \in[0,1]$ and change its position on its corner until if we reflect the colors in horizontal plane, the black and white reflected in one point, at that moment the three primery colors and its forests form cercle, starting with red as $0^{\circ}$ (see Fig. 6) [22-24].

Later on, the progress of this method was followed by specifying the brightness/value/lightness, the specified saturation of the range from 0 along the axis to 1 at the point most suitable for each pair of other parameters, however regarding this work the (Saturation, Value) $=(100 \%$, $100 \%$ ) to focus just on one level (Hue). 


\section{Results}

For the experiment, the set of random samples has been prepared. Table 3 presents the selected collection of read data, and from the table we can follow the process of color recognition. First the dataset is registered in RGB format, which is then translated to the aforementioned $M($ Max), $m$ ( $\min$ ) and Chroma values. Using Eq. (4) and Eq. (5) the system converts the data to the values of Hue, which gives us a result of degree for the given sample. This data is used then for the sorter to move the servo into the given position and practically sort out the sample according to its registered value. It is important to note, that in Table 4 the "Comment" column gives us feedback on the result as it was "compatible" or not with the previously defined color ranges.

A circular diagram is presented to show the intervals of color and some sample readings in a batch of randomly selected samples. This is shown in Fig. 7. Comparing the results of each color we note that for the blue and green the target is approximately in the middle, on the contrary in the case of the other colors the degrees are spread out more randomly along the ranges. For example the red color is detected in the edge of the logged interval.

Fig. 8 shows the average results deviating from the center of the color range, compared to the half scales of the color range. The bar chart reveals, that the average blue and green colors detection is almost in the middle of the range with small min and max deviation. The orange, yellow and red colors are further from the middle of the

Table 3 The value calculation of the matrix indicate hue, saturation and value

\begin{tabular}{lccccccc}
\hline Input color & $\mathrm{R}$ & $\mathrm{G}$ & $\mathrm{B}$ & $\mathrm{MAX}$ & $\min$ & chroma & Hue \\
\hline Red & 74 & 8 & 16 & 74 & 8 & 66 & 352 \\
Blue & 18 & 16 & 21 & 21 & 16 & 5 & 264 \\
Green & 12 & 37 & 13 & 37 & 12 & 25 & 122 \\
Yellow & 46 & 51 & 24 & 51 & 24 & 27 & 71 \\
Orange & 48 & 14 & 8 & 48 & 8 & 40 & 9 \\
Empty & 14 & 10 & 11 & 14 & 10 & 4 & 345 \\
\hline
\end{tabular}

Table 4 Comparisation of the input and output the result

\begin{tabular}{lcccc}
\hline Input color & $\begin{array}{c}\text { Result in } \\
\text { Degree }\end{array}$ & Logged & $\begin{array}{c}\text { Result } \\
\text { color }\end{array}$ & Comment \\
\hline Red & $7^{\circ}$ & {$\left[-12^{\circ}\left(348^{\circ}\right), 7^{\circ}\right]$} & red & compatible \\
Blue & $264^{\circ}$ & {$\left[180^{\circ}, 309^{\circ}\right]$} & blue & compatible \\
Green & $122^{\circ}$ & {$\left[80^{\circ}, 179^{\circ}\right]$} & green & compatible \\
Yellow & $71^{\circ}$ & {$\left[20^{\circ}, 79^{\circ}\right]$} & yellow & compatible \\
Orange & $9^{\circ}$ & {$\left[8^{\circ}, 19^{\circ}\right]$} & orange & compatible \\
Empty & $345^{\circ}$ & {$\left[310^{\circ}, 347^{\circ}\right]$} & other & compatible \\
\hline
\end{tabular}

\section{Reflection of the result colors into the Hexcone model}
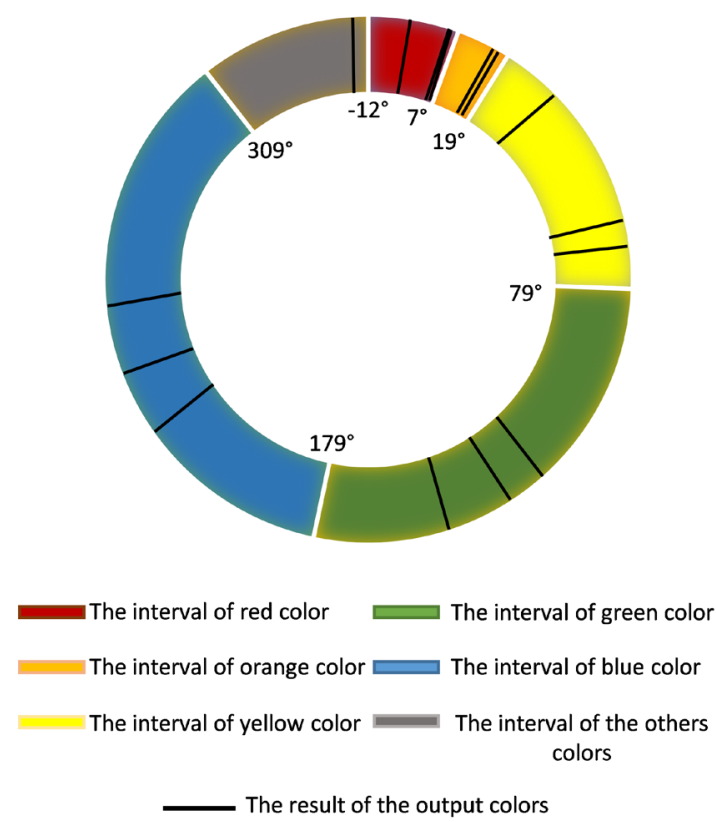

Fig. 7 Color intervals and registered measurements in a random set of samples

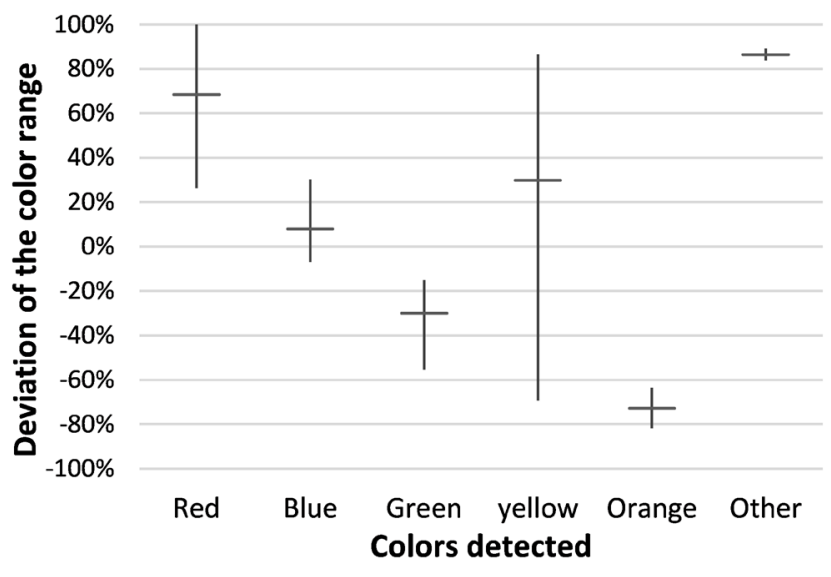

Fig. 8 The average deviation of the results from the middle of the color range

range, meaning that the detected colors were consequently off from the middle of the defined color ranges. Orange results have very small deviation, however the average is off from the center - meaning that the samples are physically off from the defined range center. The results stay in the given ranges, even with some red samples shooting to the edge of the range. The proposed solution is acceptable from the aspect of sorting automation - uncertain "other" measurement samples may be relocated to the beginning of the process and remeasured with the possibility of slightly different lighting and detection mechanisms. 
Further advantage of the application is the parallel action, giving the example of the parallel execution between the servo and the sensor due to which, we minimize time loss and speed up the candy classification remarkably.

\section{Conclusion}

To improve the productivity and the safety of food industry solutions we posed the idea of controlling food products (e.g. candies) according to its color in an cost- and time efficient way. To implement this concept, we proposed a sorter machine of the coated chocolate candy. The machine is directed by an Arduino command servo, stepper motor and color sensor TCS3200. The color sensor is able to detect the light intensity reflected from the product then combine it with the frequency of the wavelength to give us really necessary value in the RGB space, that by developing the Arduino it can be converted from the RGB space to HSL domain. After calculations based on RGB data and transforming equations the read colors will be provided in form of degrees to render it easier to know exactly to which zone of color it belongs. According to the

\section{References}

[1] Aleixos, N., Blasco, J., Navarrón, F., Moltó, E. "Multispectral inspection of citrus in real-time using machine vision and digital signal processors", Computers and Electronics in Agriculture, 33(2), pp. 121-137, 2002.

https://doi.org/10.1016/S0168-1699(02)00002-9

[2] Blasco, J., Aleixos, N., Gómez, J., Moltó, E. "Citrus sorting by identification of the most common defects using multispectral computer vision", Journal of Food Engineering, 83(3), pp. 384-393, 2007. https://doi.org/10.1016/j.jfoodeng.2007.03.027

[3] Diaz, R., Faus, G., Blasco, M., Blasco, J., Moltó, E. "The application of a fast algorithm for the classification of olives by machine vision", Food Research International, 33(3-4), pp. 305-309, 2000. https://doi.org/10.1016/S0963-9969(00)00041-7

[4] Wulfsohn, D., Sarig, Y., Algazi, R. V. "Defect sorting of dry dates by image analysis", Canadian Agricultural Engineering, 35(2), pp. 133-139, 1993.

[5] Edan, Y., Pasternak, H., Shmulevich, I., Rachmani, D., Guedalia, D., Grinberg, S., Fallik, E. "Color and Firmness Classification of Fresh Market Tomatoes", Journal of Food Science, 62(4), pp. 793-796, 1997. https://doi.org/10.1111/j.1365-2621.1997.tb15457.x

[6] Gergely, Z., Beke, J. "A micro-controller based algorythm for fast and robust edge detection in white paprika sorting process", Mechanical Engineering Letters, 10, pp. 161-169, 2013.

[7] Mohd., S. A., Mohd S. R. "Automatic multi machine operation with product sorting and packaging by their colour and dimension with speed control of motors", In: International Conference on Advances in Electrical Technology for Green Energy (ICAETGT), Coimbatore, India, 2017, pp. 88-92. https://doi.org/10.1109/ICAETGT.2017.8341466 given results, and knowing the ranges of the deviaton, the sorting can be performed.

Future paths may point to solutions make the system faster with changing the servo rotation complete turn and no need to come back from the point 360 to 0 but continue direct in the same way, also to increase the process of production we can use a conveyor belt to move the items faster under the color sensor. Precision could be improved by using filters to reduce reflectance.

The concept also shows promising future with possible network adaptation - the data from the microcontroller board could be transferred to a cost effective IoT compatible card computer, housing data, allowing remote access via the network, and allowing monitoring and possible control of the process from the far, pointing to implementations of Industry 4.0 approaches in food processing industry.

\section{Acknowledgement}

The authors would like to thank the Stipendium Hungaricum Scholarship (Miskolc, Budapest), (application ID: 166125) for the financial support.

[8] Blasco, J., Aleixos, N., Moltó, E. "Machine Vision System for Automatic Quality Grading of Fruit", Biosystems Engineering, 85(4), pp. 415-423, 2003.

https://doi.org/10.1016/S1537-5110(03)00088-6

[9] Blasco, J., Cubero, S., Gómez-Sanchís, J., Mira, P., Moltó, E. "Development of a machine for the automatic sorting of pomegranate (Punica granatum) arils based on computer vision", Journal of Food Engineering, 90(1), pp. 27-34, 2009. https://doi.org/10.1016/j.jfoodeng.2008.05.035

[10] Zhang, C., Suslick, K. S. "Colorimetric Sensor Array for Soft Drink Analysis", Journal of Agricultural and Food Chemistry, 55(2), pp. 237-242, 2007.

https://oi.org/10.1021/jf0624695

[11] Abu Shawish, H. M., Ghalwa, N. A., Saadeh, S. M., Harazeen, H. E. "Development of novel potentiometric sensors for determination of tartrazine dye concentration in foodstuff products", Food Chemistry, 138(1), pp. 126-132, 2013. https://doi.org/10.1016/j.foodchem.2012.10.048

[12] Kanade, A., Shaligram, A. "Prepackaging Sorting of Guava Fruits using Machine Vision based Fruit Sorter System based on K-Nearest Neighbor Algorithm", International Journal of Scientific Research in Computer Science, Engineering and Information Technology, 3(3), pp. 2456-3307, 2018.

[13] Rahman, M. J., Prosad Das, D., Islam, O., Zaman, H. U. "A Novel Design of a Robotic Object Sorter Based on Color Differences using Image Processing Techniques", In: International Conference on Computer, Communication, Chemical, Material and Electronic Engineering (IC4ME2), Rajshahi, Bangladesh, 2018. https://doi.org/10.1109/IC4ME2.2018.8465620 
[14] Henry, T., Laurence, Ishak, Jie, F. "Design and Construction of Color Sensor Based Optical Sorting Machine", In: $5^{\text {th }}$ International Conference on Instrumentation, Control, and Automation (ICA), Yogyakarta, Indonesia, 2017, pp. 36-40. https://doi.org/10.1109/ICA.2017.8068409

[15] Quevedo, R. A., Aguilera, J. M., Pedreschi, F. "Color of Salmon Fillets By Computer Vision and Sensory Panel", Food and Bioprocess Technology, 3(5), pp. 637-643, 2010. https://doi.org/10.1007/s11947-008-0106-6

[16] León, K., Mery, D., Pedreschi, F., León, J. "Color measurement in $\mathrm{L}^{*} \mathrm{a}^{*} \mathrm{~b}^{*}$ units from RGB digital images", Food Research International, 39(10), pp. 1084-1091, 2006. https://doi.org/10.1016/j.foodres.2006.03.006

[17] Afshari-Jouybari, H., Farahnaky, A. "Evaluation of Photoshop software potential for food colorimetry", Journal of Food Engineering, 106(2), pp. 170-175, 2011.

https://doi.org/10.1016/j.jfoodeng.2011.02.034

[18] Bordbar, M. M., Tashkhourian, J., Hemmateenejad, B. "Qualitative and quantitative analysis of toxic materials in adulterated fruit pickle samples by a colorimetric sensor array", Sensors and Actuators B: Chemical, 257, pp. 783-791, 2018.

https://doi.org/10.1016/j.snb.2017.11.010
[19] Patel, H.,Joy, R., Macwan, S., Modi, H. "IOT Color Based Object Sorting Machine", International Journal of Applied Engineering Research, 13(10), pp. 7383-7387, 2018.

[20] Goudet, L. C. "M\&M's' ${ }^{\circledR}$ Color Sorter", [pdf] Harvey Mudd College, Final Project Report, 2009. Available at: http://pages. hmc.edu/harris/class/e155/projects09/Goudet.pdf [Accessed: 04 September 2018]

[21] Tan, J., Lien, J.-M., Gingold, Y. "Decomposing Images into Layers via RGB-Space Geometry", ACM Transactions on Graphics (TOG), 36(1), pp. 7:1 - 7:14, 2017. https://doi.org/10.1145/3072959.2988229

[22] Levkowitz, H., Herman, G. T. "GLHS: A Generalized Lightness, Hue and Saturation Color Model", CVGIP: Graphical Models and Image Processing, 55(4), pp. 271-285, 1993. https://doi.org/10.1006/cgip.1993.1019

[23] Smith, A. R. "Color Gamut Transform Pairs", ACM SIGGRAPH Computer Graphics, 12(3), pp. 12-19, 1978. https://doi.org/10.1145/965139.807361

[24] Joblove, G. H., Greenberg, D. "Color spaces for computer graphics", ACM SIGGRAPH Computer Graphics, 12(3), pp. 20-25, 1978. https://doi.org/10.1145/965139.807362 